\title{
Expert system for diagnosis of womens' menstrual cycle using natural family planning method
}

\author{
Andrzej Urbaniak \\ Institute of Computing Science, Poznań University of Technology \\ ul. Piotrowo 3A, 60-965 POZNAŃ, Poland \\ Phone: +4861 790790; fax: +4861 771525; \\ E-mail: urbaniak@pozn1v.put.edu.pl
}

\begin{abstract}
In the contribution the algorithm and user-friendly software for fertility diagnosis, are considered. A part of research concerning the algorithms for multi-check methods was presented by Urbaniak (1994a). In this paper will be present the algorithms for expert system supporting the women fertility diagnosis and evaluation of pregnancy achievement.

\section{Keywords}

Biological system, expert system, Natural Family Planning (NFP), medical diagnosis
\end{abstract}

\section{INTRODUCTION}

People have been interested more and more in ecological aspects of life. This is connected to the application of any natural methods in human life, as well in normal as in sick conditions. These tendencies cause the great interest in natural methods determining fertility and infertility days in the woman menstrual cycles. Methods of Natural Family Planning (NFP) are interesting because of their high effectiveness, comparable to hormone contraception methods. following:

Some may complain of two inconveniences of the NFP methods. These are the

- necessity of daily observations and documentation's of fertility symptoms,

- necessity of well education to determine fertility and infertility days.

\footnotetext{
* Presented on 17th IFIP Conference on System Modelling and Optimization, July 10-14, Prague
} 
In many scientific centres research for easier NFP methods application is provided. There are two main approaches. The first one, it is construction of special devices to measure hormone changes during the woman cycle. This research has been developed, for example, by Thornton et al.(1990), Brown et al. $(1984,1992)$. The enzyme immunoassays for urinary oestrogen detection was elaborated and effectively used to determine fertility days. The method was worked out as a simply procedure which can be performed at home.

Second approach is based on the microprocessor technic. BIOSELF system is first, fully acceptable device. It is an electronic thermometer with the microcomputer (Flynn \&Brooks, 1990). This system was programmed according to Temperature-only Method and had been tested in many countries.

Our research it is the second approach which uses standard PC computers with Expert System (ES) to create fertility awareness. The advantage of PC systems application is: possibility of determining fertile days without education about the rules of NFP methods. Of course, the most important is constant observation of woman body symptoms. It is part of the activity in which woman can not be replaced. The rest-part, with rules of NFP methods, takes over the PC software. The software prepared as a user-friendly program is the crucial point of the system.

\section{BASES FOR NFP METHODS}

Natural Family Planning methods are based on three major facts.

1. There is only one ovulation in the cycle. There is possibility for the second ovum to grow up, but only during the next 24 hours to the first ovulation. Ogino and Knaus have described that ovulation in the menstrual cycle occurs about $14 \pm 2$ days before the next menstruation.

2. The ovum life time is no longer than 24 hours. The possibility for the conception is usually shorter.

3. The sperm life in the women's vagina is maximum 5 to 6 days. This time depends on the special kind of cervix mucus which is necessary to keep the sperm alive 5-6 days.

General conclusions is that conception may occur in the time around ovulation and no longer than during 6 to 7 days in the cycle. During other days of the cycle conception is not possible.

Women menstrual cycle is controlled by the complex interaction of hormones (the chemical messengers of the body) and reproductive organs. This causes that each month a grown up ovum is released from the ovary.

Thus the women menstrual cycle consists of the following phases.

Phase 1 it is time from first day of menstruation to the moment when follicles start to grow up in the ovaries. As length of this part of the cycle varies from cycle to cycle of the individual women, as well as from women to woman, it is sometimes difficult to of make accurate assessments of the beginning of fertility. For this reason it is called as relatively infertile phase.

Phase 2 starts from the time when follicles begin to grow up to first signs of any mucus or wetness to 48 hours after to few days after ovulation (time calculated by temperature and mucus observations). (It together takes 24 hours for the lifespan of the ovum, 5-6 days for the livespan of the sperm, because it is impossible to be precise about the actual time of ovulation.) This is fertile phase. 
Phase 3 is counted from a few days after ovulation (calculated by temperature and mucus observations) until the last day before the next menstruation which is the end of that cycle. This is called absolutely infertile phase.

From Ogino and Knaus discovery a lot of NFP methods have been worked out.. Now a lot of these methods have been documented by the wide experimental research provided by world famous scientific centres.

\section{MODERN NFP METHODS}

Calendar (or Calculation) Method was the earliest method of NFP. This method is based on the fact, that ovulation occurs $14+2$ days before the next menstruation. Taking into account the "worst case" (the length of the shortest cycle from the last 12 cycles) the fertility days are calculated according to the simple rule. In the thirties this method was very the important for many couples. In those years women had more regular cycles than now, because they did not work professionally, and ecological conditions were better. Thus then the effectiveness of the Calendar Method was high too. Presently, it is impossible to based on the regularity of women's cycle. Many factors of present life conditions cause irregularity of women's cycles. In consequence, the determination of the fertility or infertility days in women cycles can be possible only when using the every day observations of major fertility signs.

There are the following:

1. basal body temperature (BBT),

2. quantity and quality of the cervix mucus,

3. state of the cervix.

The modern methods of fertility awareness apply one or more of mentioned above signs. Daily observation of these signs gives with the high reliability, possibility to determining fertile and infertile days.

\subsection{Temperature-only Method}

This method is based on the fact, that woman's basal body temperature(BBT) rises suddenly after the ovulation. The minimal shift of the temperature has to be 0.2 Celsius degrees, and the fertile phase ends when the basal body temperature elevates at least $0.2{ }^{\circ} \mathrm{C}$ for three consecutive days, without any disturbances. The Temperature-only Method allows to determine only post-ovulatory infertile phase. BBT to be used in this method, and in (any other methods with BBT, has to be taken for five or eight minutes at the same waking time each morning. Rules for the Temperature-only Method were presented by Marshal. A lot of researchers underline the high effectiveness of the Temperature-only Method (Donnay, 1991).

\subsection{Billings Ovulation Method}

The cervical mucus method of NFP relies on the woman self observation of presence and changes cervical mucus throughout the menstrual cycle, and these changes are used to determine fertile and infertile phases. This method is also known as the Billings Ovulation Method or Ovulation Method Billings (Brown et al., 1984, 1987). Two important factors are 
needed: sensation and appearance of the mucus. The important point is that the cervix produces different types of mucus - fertile-type mucus and infertile-type mucus which appear at different parts of the cycle. Together with ovum growing up in the ovary, the cervical mucus becomes more clear, stretchy, wet and slippy. It depends on rising level of oestrogen. After ovulation the mucus becomes thicker, sticky or disappear - dry days.

\subsection{Double-check Method (Sympto-Thermal Method)}

The sympto-thermal double-check method is based on the observation of three major signs of the menstrual cycle. These are the following:

1. basal body temperature (BBT);

2. quantity and quality of the mucus;

3. state of the cervix

All changes of these signs are caused by the changes of hormones levels in the blood. From the technical point of view it would be very interesting to measure precisely these hormones levels. But the three signs, mentioned above, are able to measure these parameters indirectly.

Historically, the Sympto-Thermal Method is based on the earlier worked out methods. In general, all of these methods of NFP can be divided into two groups. First one - it is group of the one-symptom methods based on: either the BBT, either the mucus, or the cervix. Second group there is group of the methods which apply two and more fertility signs to crosscheck beginning and end of fertile phase. This group has been intensively developed, especially in the last two decades. The Sympto-Thermal Methods (Double-Check Method) have one of the highest effectiveness rate. Formula devised by Raymond Pearl in the 1930's is standard procedure expressing the effectiveness of any NFP Methods.

The particular rules of the Sympto-Thermal Method depend on the following factors (Flynn\&Brooks, 1990):

1. status of women life (ordinary cycle, post-partum cycle, premenopause cycle, and post-pill cycle);

2. length of the history of observations,

3. sort and number of signs observed.

These different rules must be implemented in the engine module of the Expert System. The women needs to declare properly observed mucus sign concerning its sensation (feeling) and appearance. In the similar way the women needs to check and describe proper features of the cervix (position, opening, firmness). Taking into account every day observation of the above two signs and adding the BBT results we obtain sufficient data to estimate fertility stage on every day of women menstrual cycle.

From the algorithm point of view, there is very important to define the temperature shift. Temperature shift is connected to the ovulation of the ovulation and is described as a higher BBT level sustaining for at least three consecutive days without any disturbances. . Additionally, to recognize temperature shift, the cover line needs to be drawn over the low temperature phase (Flynn\&Brooks, 1990).

For the mucus and cervix observation, the peak day is defined as a last day of the fertile-type mucus or maximum cervix features. All rules of sympto-thermal method are given by Flynn \& Brooks, (1990). 


\section{CHARACTERISTICS OF AN EXPERT SYSTEM}

We must note that systematic and responsible observations are very important factors for adaptation of the NFP methods. This remark especially important when collection of data and chart interpretation is realized automatically using a PC system.

The computer system can fulfil the following expectations concerning with the two main inconveniences of NFP methods:

- necessity of documentation of daily observations,

- good knowledge about the rules of fertility and infertility to determine.

These above mentioned inconveniences can be realized using the well designed DSS or ES with clear and friendly graphical interface (Urbaniak, 1994c). The user ought to only introduce to the system the observed signs of fertility and other functions are realized by algorithms. All data are introduced by the way of answers for the questions which are highlighted on the screen.

There are the following advantages in the well-designed Expert System: the increase availability, the cost reducing, the danger reducing, the permanence, the multiple expertise, the reliability increasing, the explanation, the fast response, the steady, unemotional, and complete response at all times, the intelligent tutor, the intelligent database.

The worked out first implementations of the algorithms concern the basic situations in the woman live only. The special cases demands the different rules to introduce into the algorithms. A study on this problem are begun now (Burdziak, 1994).

\section{USER INTERFACES}

\subsection{BILMETH - Billings Ovulation Method}

The worked out friendly user's interface allows to apply the system without special experience in the computer science (Szczepaniak, 1994). There are following rules of the options (Fig.1):

1. Choice of the accessible option is realized by pressing the "hot" key, identified by the highlighting letter or by mouse. This change is presented graphically by simulation of the pressed key.

2. The program has help function. This function is connected with given operation which is under realization.

3. There is unification way of data introducing. All data must be confirmed by Enter

\section{Options}

Menu USER. This option enables to create a new user (NEW) or register of an existing user (PRESENT). The each individual is identified by forename, name and password.

Menu NEW. This instruction is used to create new chart of the cycle observation. The important data about each cycle is at beginning. Additionally we can shortly describe the special feature of the cycle (optionally).

Menu PRESENT. This instruction is prepared to introduce of data the present cycle. The user has to choose proper symptom from given set the and confirm it.

Menu HIST is used to analyze previous cycles (history of the cycles).

Menu $d R U K$ enables to print out the cycle chart. 
Menu HELP and INFO there is context help to apply system and gives information about authors and institution.

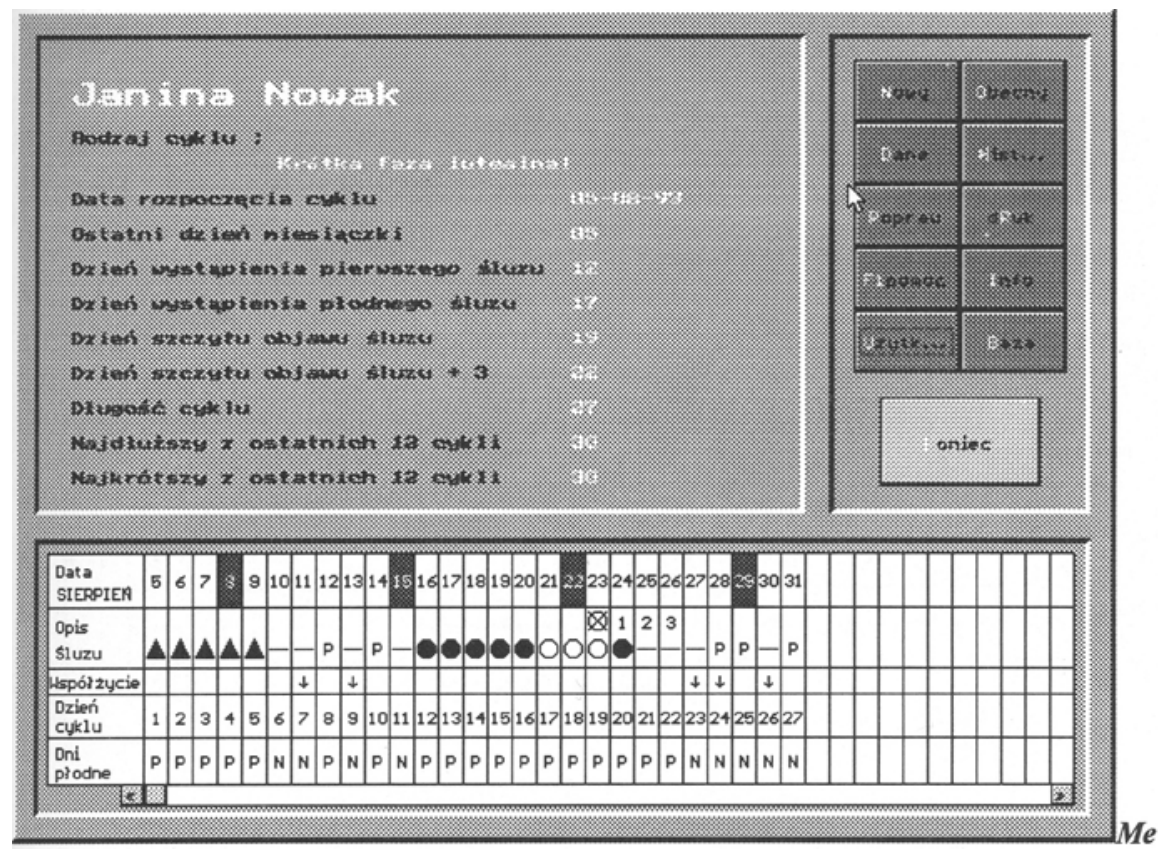

Fig. 1 An example of dialogue window for Billings Ovulation Method

\subsection{STBB - Double-check Method}

The elaborated software is friendly to the user who knows only basic rules of the keyboard and how to use mouse (Białobłocki, Urbaniak, 1994, Urbaniak, 1994b).

During working phase, the screen is divided into three parts (Fig. 2):

- menu line (first screen line);

- status line (last screen line);

- work part (between menu and status lines).

Menstrual Cycle Chart Creation. Spreading of the CHART menu allows to introduce new chart user or to fulfil existing chart. The user can choose of any chart cycle identified by date of its preparation.

Mark Data On the Chart. All observations are marked in days only. It is impossible to edit a day which edition was finished, nor any of the future days. Edition of each day can be finished or closed. The difference between closed and finished concern the situation when all symptoms are observed already or only one of them. After when the day edition is finishing no changes are possible. In situation when some symptoms were not observed and marked we resign edit it. In the window with the mucus sensation is presented.

Chart Printing. An option allows to print out any chart of menstrual cycle. 


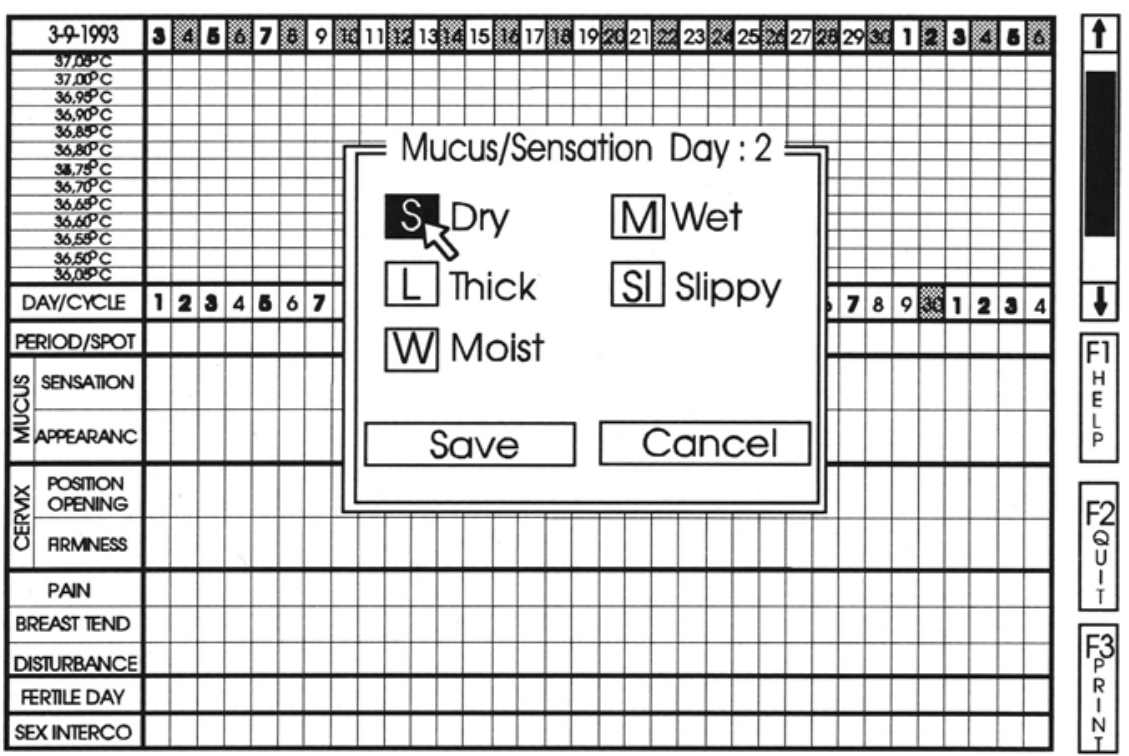

Fig. 2. An example of the dialogue window

\section{FUTURE RESEARCH}

In many scientific centres research on NFP methods is performed and new methods and modern modification of an existing ones are published. The intensive research expansion in the computer science and especially in the field of expert systems and artificial intelligent allows to apply these results to software systems. The software presented above is based on the scientific background of NFP and now, the software is tested by NFP teachers.

Our next research plans concerns following directions.

First one concern supplementation of the software with rules of the special situations. And after this we will have the basic software product, but only for main situation of the woman life. In the next step we plan to extend the software to the special situations: i.e. after birth, premenopause and post hormonal contraception. These special situations demand the modification of the rules and algorithm to determine fertility and infertility days.

Second direction concerns creation of the software for computer oriented teaching. Presently we have first version of the software which actually has been tested for different NFP methods.

NFP effectiveness study this is third direction. All prepared software generate formatted files with necessary for effectiveness calculations data, according to European NFP Study (Urbaniak, Zmyślony, 1994).

The important results of the research were achieved thanks to good collaboration between computer science engineers and NFP teachers, and feedback under the form of well organized testing procedure. 


\section{REFERENCES}

Białobłocki, T., Urbaniak, A. (1994) Fertility analysis in the woman cycle using symptothermal method. Biulletin of Polish National Society of Natural Family Planning Teachers, 3, 2-4. (in polish)

Brown, J.B., Blackwell, L.F., Billings, J.J. et al. (1984) Determination of ovarian hormone levels in urine for identifying the fertile and infertile phases of the cycle. Family Health Internat. Expert Meeting, North Carolina.

Brown, J.B., Blackwell, L.F., Billings, J.J. et al. (1987) Natural family planning. American Journal of Obstetrics Gynecology, 157, 1082-9.

Brown, J.B., Blackwell, L.F., Holmes, J., Smyth, K. (1992) New assays fir identifying the fertile period. International Journal of Gynecology \& Obstetrics, 1, (1).

Burdziak, A. (1994) NFP - expert system. Master Degree, Institute of Comp. Science, Poznań University.

Donnay, F. (1991) Maitrese de la fecondite. L'enfant en milien tropical, Centre Internationale de l'Enfance, no. 193-194, Paris.

Flynn, A., Brooks, M. (1990) A manual of Natural Family Planning. Unwin Hyman Limited, London.

Szczepaniak, P. (1994) DSS for ovulation method. Master degree, Institute of Comp. Science.

Thornton, S.J., Pepperell, R.J. Brown, J.B. (1990) Home monitoring of gonadotropin ovulation induction using the Ovarian Monitor. Fertility and Sterility, 56, (6), 107682 .

Urbaniak, A. (1994a) Computer and Natural Family Planning. Biulletin of Polish National Society of Natural Family Planning Teachers, 2, 6-7. (in polish)

Urbaniak, A. (1994b) Fertility diagnosis and simulation based on the multi-check method of natural family planning, in Proc. CISS - First Joint Conf. of International Simulation Societas (eds. J. Halin, W. Karplas), Zurich.

Urbaniak, A. (1994c) Main features of the expert systems for determining the fertile and infertile days in the women menstrual cycle. Plenary lecture on VI Congress IFFLP/FIDAF, Lublin, Poland.

Urbaniak, A., Zmyślony, R. (1994) An effectiveness research of NFP methods using NPR system, in VI Congress IFFLP/FIDAF Lublin (ed. R. Sikorski), Poland. 